\title{
HAWQ: Hessian AWare Quantization of Neural Networks with Mixed-Precision
}

\author{
Zhen Dong*, Zhewei Yao*, Amir Gholami*, Michael W. Mahoney, Kurt Keutzer \\ University of California, Berkeley \\ \{zhendong, zheweiy, amirgh, mahoneymw, and keutzer\}aberkeley.edu
}

\begin{abstract}
Model size and inference speed/power have become a major challenge in the deployment of Neural Networks for many applications. A promising approach to address these problems is quantization. However, uniformly quantizing a model to ultra low precision leads to significant accuracy degradation. A novel solution for this is to use mixed-precision quantization, as some parts of the network may allow lower precision as compared to other layers. However, there is no systematic way to determine the precision of different layers. A brute force approach is not feasible for deep networks, as the search space for mixed-precision is exponential in the number of layers. Another challenge is a similar factorial complexity for determining block-wise fine-tuning order when quantizing the model to a target precision. Here, we introduce Hessian AWare Quantization (HAWQ), a novel secondorder quantization method to address these problems. HAWQ allows for the automatic selection of the relative quantization precision of each layer, based on the layer's Hessian spectrum. Moreover, HAWQ provides a deterministic fine-tuning order for quantizing layers, based on second-order information. We show the results of our method on Cifar-10 using ResNet20, and on ImageNet using Inception-V3, ResNet50 and SqueezeNext models. Comparing HAWQ with state-of-the-art shows that we can achieve similar/better accuracy with $8 \times$ activation compression ratio on ResNet20, as compared to DNAS [36], and up to $1 \%$ higher accuracy with up to $14 \%$ smaller models on ResNet50 and Inception-V3, compared to recently proposed methods of RVQuant [26] and HAQ [35]. Furthermore, we show that we can quantize SqueezeNext to just $1 \mathrm{MB}$ model size while achieving above $68 \%$ top 1 accuracy on ImageNet.
\end{abstract}

\section{INTRODUCTION}

There has been a significant increase in the computational resources required for Neural Network (NN) training and inference. This is mainly due to larger input sizes (e.g., higher image resolution) as well as larger $\mathrm{NN}$ models requiring more FLOPs and significantly larger memory footprint. For example, in 1998 the state-of-the-art NN was LeNet-5 [19] applied to MNIST dataset with an input image size of $1 \times 28 \times 28$. Twenty years later, a common benchmark dataset is ImageNet, with an input resolution that is $200 \times$ larger than MNIST, and with NN models that have orders of magnitude higher memory footprint.

In fact, ImageNet resolution is now considered "small" for many applications such as autonomous driving where input resolutions are significantly larger (more than $40 \times$ in certain cases).

This combination of larger models and higher resolution images has created a major challenge in the deployment of NNs in application environments with computationally constrained

\footnotetext{
${ }^{*}$ Equal contribution.
}

resources such as surveillance systems or ADAS systems in passenger cars. This trend is going to accelerate further in the near future.

There has been a significant effort taken by many researchers to address these issues. These could be broadly categorized as follows. (i) Finding NNs that provide the required accuracy, while remaining compact by design (i.e., with small memory footprint) and which require relatively small FLOPs. SqueezeNet [15] was an early effort here, followed by more efficient NNs such as [31], [21]. (ii) Co-designing NN architecture and hardware together. This can allow significant speed ups and savings in power consumption of the hardware without losing accuracy. SqueezeNext [7] is an example work here where the neural network and associated accelerator are codesigned. (iii) Pruning redundant filters of NN layers. Seminal works here are [9], [25], [20], [22]. (iv) Applying AutoML for both hardware aware NN design as well as quantization. Notable works here are DNAS [36] and HAQ [35]. (v) Using quantization (reduced precision) instead of float or double precision, which can significantly speed up inference time and reduce power consumption. This paper exclusively focuses on quantization, but other approaches could be used in conjunction of our method to allow for further possible reduction on the model size.

Quantization needs to be performed for both NN parameters (i.e., weights) as well as the activations to reduce the total memory footprint of the model during inference. However, the main challenge here is that a naïve quantization can lead to significant loss in accuracy. In particular, it is not possible to reduce the number of bits of all weights/activations of a general convolutional network to ultra low-precision without significant accuracy loss. This is because not all the layers of a convolutional network allow the same quantization level. A possible approach to address this is to use mixedprecision quantization, where higher precision is used for certain "sensitive" layers of the network, and lower precision for "non-sensitive" layers. However, the search space for finding the right precision for each layer is exponential in the number of layers. Moreover, to avoid accuracy loss we need to perform fine-tuning (i.e. re-training) of the model. As we will discuss below, quantizing the whole model at once and then finetuning is not optimal. Instead, we need to perform multi-stage quantization, where at each stage parts of the network are quantized to low-precision followed by quantization-aware fine-tuning to recover accuracy. However, the search space 

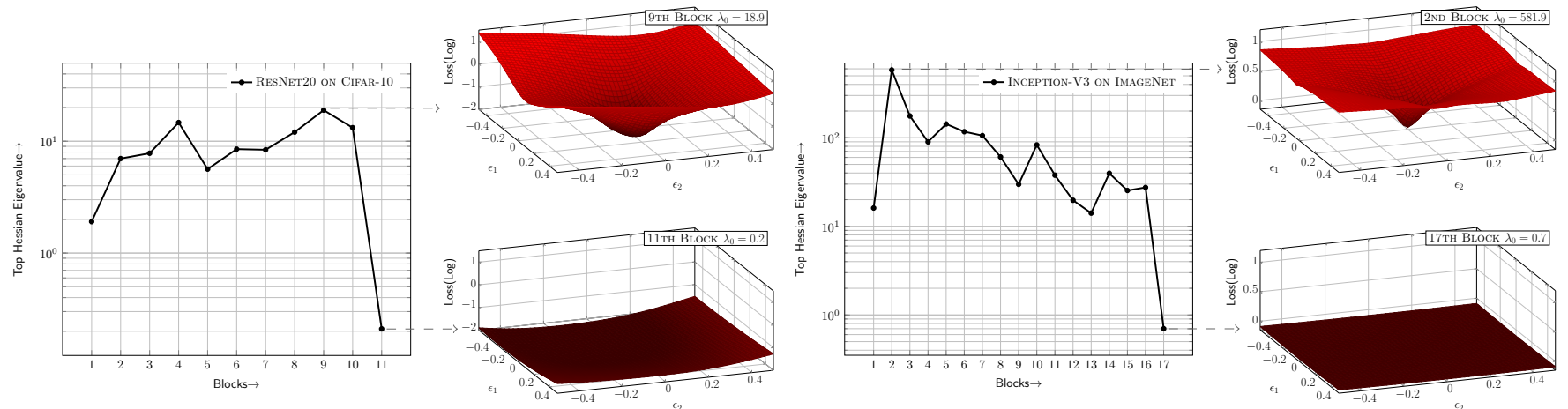

Fig. 1: Top eigenvalue of each individual block of pre-trained ResNet20 on Cifar-10 (Left), and Inception-V3 on ImageNet (Right). Note that the magnitudes of eigenvalues of different blocks varies by orders of magnitude. See Figure 6 and 7 in appendix for the $3 D$ loss landscape of other blocks.

to determine which layers to quantize first is factorial in the number of layers. In this paper, we propose a Hessian guided approach to address these challenges. In particular, our contributions are the following.

1) The search space for choosing mixed-precision quantization is exponential in the number of layers. Thus, we present a novel, deterministic method for determining the relative quantization level of layers based on the Hessian spectrum of each layer.

2) The search space for quantization-aware fine-tuning of the model is factorial in the number of blocks/layers. Thus, we propose a Hessian based method to determine fine-tuning order for different $\mathrm{NN}$ blocks.

3) We perform ablation study of HAWQ, and we present novel quantization results using ResNet20 on Cifar10, as well as Inception-V3/ResNet50/SqueezeNext on ImageNet. Comparison with state-of-the-art shows that our method achieves higher precision (up to $1 \%$ ), smaller model size (up to $20 \%$ ), and smaller activation size (up to $8 \times$ ).

The paper is organized as follows. First, in $\S$ II we will discuss related works on model compression. This is followed by describing our method in $\S$ IIII and our results in $\S$ IV. Finally, we present ablation study in $\S \mathrm{V}$, followed by conclusions.

\section{RELATED WORK}

Recently, significant efforts have been spent on developing new model compression solutions to reduce the parameter size as well as computational complexity of NNs [4], [8], [11], [28], [5], [42], [17], [13], [3], [41]. In [9], [22], [20], pruning is used to reduce the number of non-zero weights in NN models. This approach is very useful for models that have very large fully connected layers (such as AlexNet [18] or VGG [33]).

For instance, the first fully-connected layer in VGG-16 occupies $408 \mathrm{MB}$ alone, which is $77.3 \%$ of total model size. Large fully-connected layers have been removed in other fully convolutional networks such as ResNet [10], or Inception family [34].

Knowledge distillation introduced in [11] is another direction for compressing NNs. The main idea is to distill information from a pre-trained, large model into a smaller model. For instance, it was shown that with knowledge distillation it is possible to reduce model size by a factor of 3.6 with an accuracy of $91.61 \%$ on Cifar-10 [30].

Another fundamental approach has been to architect models which are, by design, both small and hardware-efficient. An initial effort here was SqueezeNet [15] which could achieve AlexNet level accuracy with $50 \times$ smaller footprint through network design, and additional $10 \times$ reduction through quantization [8], resulting in a $\mathrm{NN}$ with $500 \times$ smaller memory footprint. Other notable works here are [13], [31], [41], [21], [3], where more accurate networks are presented. Another work here is SqueezeNext [7], where a similar approach is taken, but with co-design of both hardware architecture along with a compact NN model.

Quantization [1], [4], [28], [42], [43], [2], [40] is another orthogonal approach for model compression, where lower bit representation are used instead of redesigning the NN. One of the major benefits of quantization is that it increases a NN's arithmetic intensity (which is the ratio of FLOPs to memory accesses). This is particularly helpful for layers that are memory bound and have low arithmetic intensity. After quantization, the volume of memory accesses reduces, which can alleviate/remove the memory bottleneck.

However, directly quantizing NNs to ultra low precision may cause significant accuracy degradation.

One possibility to address this is to use Mixed-Precision quantization (MP) [36], [44]. A second possibility, Multi-Stage Quantization (MSQ), is proposed by [42], [6]. MP and MSQ can improve the accuracy of quantized NNs, but face an exponentially large search space. This is a major problem that has not been addressed in existing literature for quantization. Applying existing methods require often ad-hoc rules to choose precision of different layers which are problem/model specific and do not generalize. The goal of our work here is to address this challenge using second-order information.

\section{Methodology}

Assume that the $\mathrm{NN}$ is partitioned into $b$ blocks denoted by $\left\{B_{1}, B_{2} \ldots, B_{b}\right\}$, with learnable parameters 

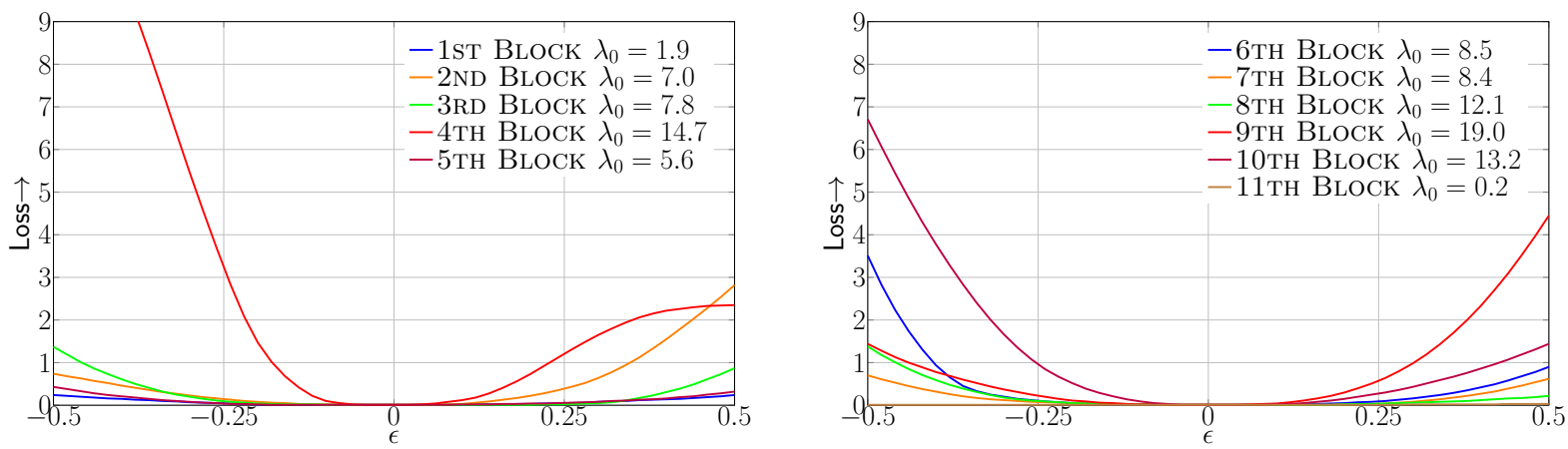

Fig. 2: 1-D loss landscape for different blocks of ResNet20 on Cifar-10. The landscape is plotted by perturbing model weights along the top Hessian eigenvector of each block, with a magnitude of $\epsilon$ (i.e., $\epsilon=0$ corresponds to no perturbation).

$\left\{W_{1}, W_{2}, \ldots, W_{b}\right\}$. A block can be a single/multiple layer(s) (or a single/multiple residual block(s) for the case of residual networks). For a supervised learning framework, the loss function $L(\theta)$ is:

$$
L(\theta)=\frac{1}{N} \sum_{i=1}^{N} l\left(x_{i}, y_{i}, \theta\right)
$$

where $\theta \in \mathbb{R}^{d}$ is the combination of $\left\{W_{1}, W_{2}, \ldots, W_{b}\right\}$, and $l(x, y, \theta)$ is the loss for a datum $(x, y) \in(X, Y)$. Here, $X$ is the input set, $Y$ is the corresponding label set, and $N=|X|$ is the size of the training set.

The training is performed by solving an Empirical Risk Minimization problem, to find the optimal model parameters. This process is typically performed in single precision, where both the weights and activations are stored with 32-bit precision.

After the training is finished, each of these blocks will have a specific distribution of floating point numbers for both the parameters, $\theta$, as well as input/output activations. For quantization, we need to restrict these floating numbers to a finite set of values, defined by the following function:

$$
Q(z)=q_{j}, \quad \text { for } z \in\left(t_{j}, t_{j+1}\right],
$$

where $\left(t_{j}, t_{j+1}\right]$ denotes an interval in the real numbers $\left(j=0, \ldots, 2^{k}-1\right), k$ is the quantization bits, and $z$ is either an activation or the weights. This means that all the values in the range of $\left(t_{j}, t_{j+1}\right]$ are mapped to $q_{j}$. In the extreme case of binary quantization $(k=1), Q(z)$ is basically the sign function. For cases other than binary quantization, the choice of these intervals can be important. One popular option is to use a uniform quantization function, where the above range is equally split [43], [14]. However, it has been argued that (i) not all layers have the same distribution of floating point values, and (ii) the network can have significantly different sensitivity to quantization of each layer. To address the first issue, different quantization schemes such as uniformly discretizing logarithmic-domain have been proposed [24]. However, this does not completely address the sensitivity problem. A sensitive layer cannot be quantized to the same level as a non-sensitive layer.

One possible approach that can be used to measure quantization sensitivity is to use first-order information, based on the gradient vector. However, the gradient can be very misleading. This can be easily illustrated by considering a simple 1-d parabolic function of the form $y=\frac{1}{2} a x^{2}$ at origin (i.e., $x=0$ ). The gradient signal at the origin is zero, irrespective of the value of $a$. However, this does not mean that the function is not sensitive to perturbation in $x$. We can get a better metrics for sensitivity by using second-order information, based on the Hessian matrix. This clearly shows that higher values of $a$ result in more sensitivity to input perturbations.

For the case of high dimensions, the second order information is stored in the Hessian matrix, of size $n_{i} \times n_{i}$ for each block. For this case, we can compute the eigenvalues of the Hessian to measure sensitivity, as described next.

\section{A. Second-Order Information}

We compute the eigenvalues of the Hessian (i.e., the secondorder operator) of each block in the network. Note that it is not possible to explicitly form the Hessian since the size of a block (denoted by $n_{i}$ for $\mathrm{i}^{\text {th }}$ block) can be quite large. However, it is possible to compute the Hessian eigenvalues without explicitly forming it, using a matrix-free power iteration algorithm [39], [23], [38]. This method requires computation of the so-called Hessian matvec, which is the result of multiplication of the Hessian matrix with a given (possibly random) vector $v$. To illustrate how this can be done for a deep network, let us first denote $g_{i}$ as the gradient of loss $L$ with respect to the $i^{t h}$ block parameters,

$$
g_{i}=\frac{\partial L}{\partial W_{i}} .
$$

For a random vector $v$ (which has the same dimension as $g_{i}$ ), we have:

$$
\frac{\partial\left(g_{i}^{T} v\right)}{\partial W_{i}}=\frac{\partial g_{i}^{T}}{\partial W_{i}} v+g_{i}^{T} \frac{\partial v}{\partial W_{i}}=\frac{\partial g_{i}^{T}}{\partial W_{i}} v=H_{i} v,
$$

where $H_{i}$ is the Hessian matrix of $L$ with respect to $W_{i}$. Note that the second equality above, comes from the fact that $v$ is independent of $W_{i}$. We can then use power-iteration method to compute the top eigenvalue of $H_{i}$, as shown in Algorithm 1. Intuitively the algorithm requires multiple evaluations of the Hessian matvec, which can be computed using Eq. 4 


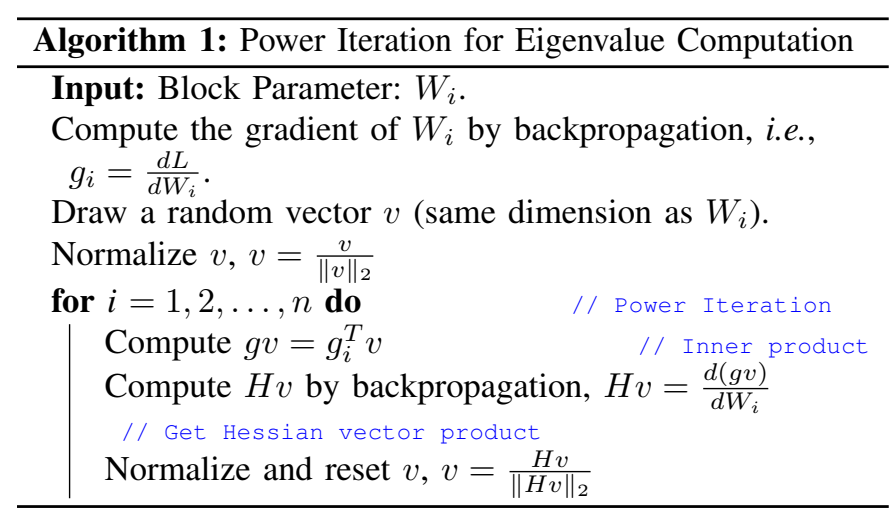

It is well known, based on the theory of Minimum Description Length (MDL), that fewer bits are required to specify a flat region up to a given threshold, and vice versa for a region with sharp curvature [29], [12]. The intuition for this is that the noise created by imprecise location of a flat region is not magnified for a flat region, making it more amenable to aggressive quantization. The opposite is true for sharp regions, in that even small round off errors may be amplified. Therefore, it is expected that layers with higher Hessian spectrum (i.e., larger eigenvalues) are more sensitive to quantization. The distribution of these eigenvalues for different blocks are shown in Figure 1 for ResNet20 on CIFAR-10 and Inception-V3 on ImageNet. As one can see, different blocks exhibit orders of magnitude difference in the Hessian spectrum. For instance, ResNet20 is an order of magnitude more sensitive to perturbations to its $9^{\text {th }}$ block, than its last block.

To further illustrate this, we provide 1D visualizations of the loss landscape as well. To this end, we first compute the Hessian eigenvector of each block, and we perturb each block individually along the eigenvector and compute how the loss changes. This is illustrated in Figure 2 and 3 for ResNet20 (on Cifar-10) and Inception-V3 (on ImageNet), respectively. It can be clearly seen that blocks with larger Hessian eigenvalue (i.e., sharper curvature) exhibit larger fluctuations in the loss, as compared to those with smaller Hessian eigenvalue (i.e., flatter curvature). A corresponding 3D plot is also shown in Figure 1 where instead of just considering the top eigenvector, we also compute the second top eigenvector and visualize the loss by perturbing the weights along these two directions. These surface plots are computed for the $9^{\text {th }}$ and last blocks of ResNet20, as well as 2nd and last blocks of Inception-V3 (the loss landscape for other blocks is shown in Figure 6 and Figure 7 .

\section{B. Algorithm}

We approximate the Hessian as a block diagonal matrix, scaled by its top eigenvalue, $\lambda$, as $\left\{H_{i} \approx \lambda_{i} I\right\}_{i=1}^{b}$. Based on the MDL theory, layers with large $\lambda$ cannot be quantized to ultra low precision without significant perturbation to the model. Thus we can use the Hessian spectrum of each block to sort the different blocks and perform less aggressive quantization to layers with large spectrum. However, some of these blocks may contain very large number of parameters, and using higher bits

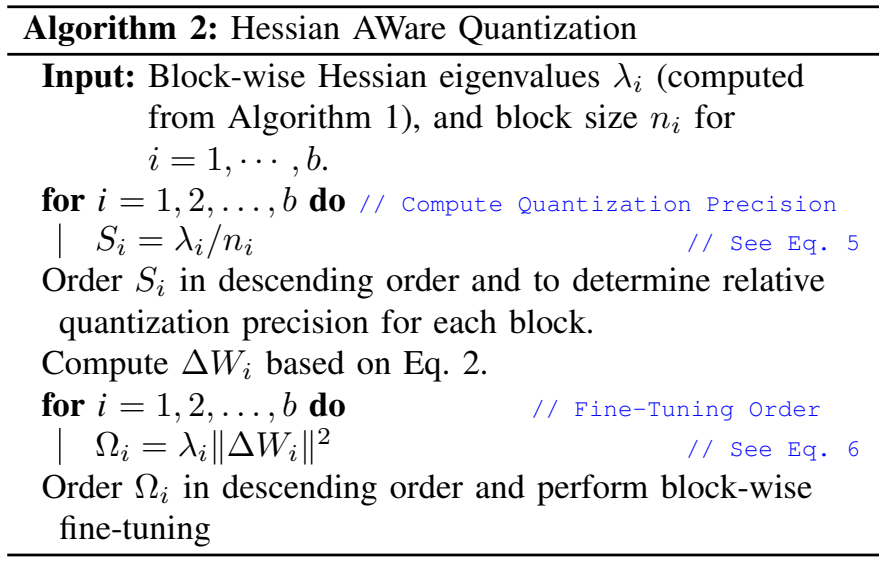

here would lead to large memory footprint of the quantized network. Therefore, as a compromise, we weight the spectrum with block's memory footprint and use the following metric for sorting the blocks:

$$
S_{i}=\lambda_{i} / n_{i},
$$

where $\lambda_{i}$ is the top eigenvalue of $H_{i}$. Based on this sorting, layers that have large number of parameters and have small eigenvalue would be quantized to lower bits, and vice versa. That is, after $S_{i}$ is computed, we sort $S_{i}$ in descending order and use it as a metric to determine the quantization precision ${ }^{1}$

Quantization-aware re-training of the neural network is necessary to recover performance which can sharply drop due to ultra-low precision quantization. A straightforward way to do this is to re-train (hereafter referred to as fine-tune) the whole quantized network at once. However, as we will discuss in $\S \mathrm{IV}$ this can lead to sub-optimal results. A better strategy is to perform multi-stage fine-tuning. However, the order in multi-stage tuning is important and different ordering could lead to very different accuracies.

We sort different blocks for fine-tuning based on the following metric:

$$
\Omega_{i}=\lambda_{i}\left\|Q\left(W_{i}\right)-W_{i}\right\|_{2}^{2},
$$

where $i$ refers to $i^{\text {th }}$ block, $\lambda_{i}$ is the Hessian eigenvalue, and $\left\|Q\left(W_{i}\right)-W_{i}\right\|_{2}$ is the $L_{2}$ norm of quantization perturbation. The intuition here is to first fine-tune layers that have high curvature as well as large number of parameters which cause more perturbations after quantization. Note that the latter metric depends on the bits used for quantization and is not a fixed metric. (See Table V] in appendix, where we show how this metric changes for different quantization precision.) The motivation for choosing this order is that fine-tuning blocks with large $\Omega_{i}$ can significantly affect other blocks, thus making prior fine-tuning of layers with small $\Omega_{i}$ futile.

\section{RESULTS}

In this section, we first present our quantization results for ResNet20 on Cifar-10, and then we present our results

\footnotetext{
${ }^{1}$ Note that, as mentioned in the limitations section, $S_{i}$ does not give us the exact bit precision but a relative ordering for the bits of different blocks.
} 

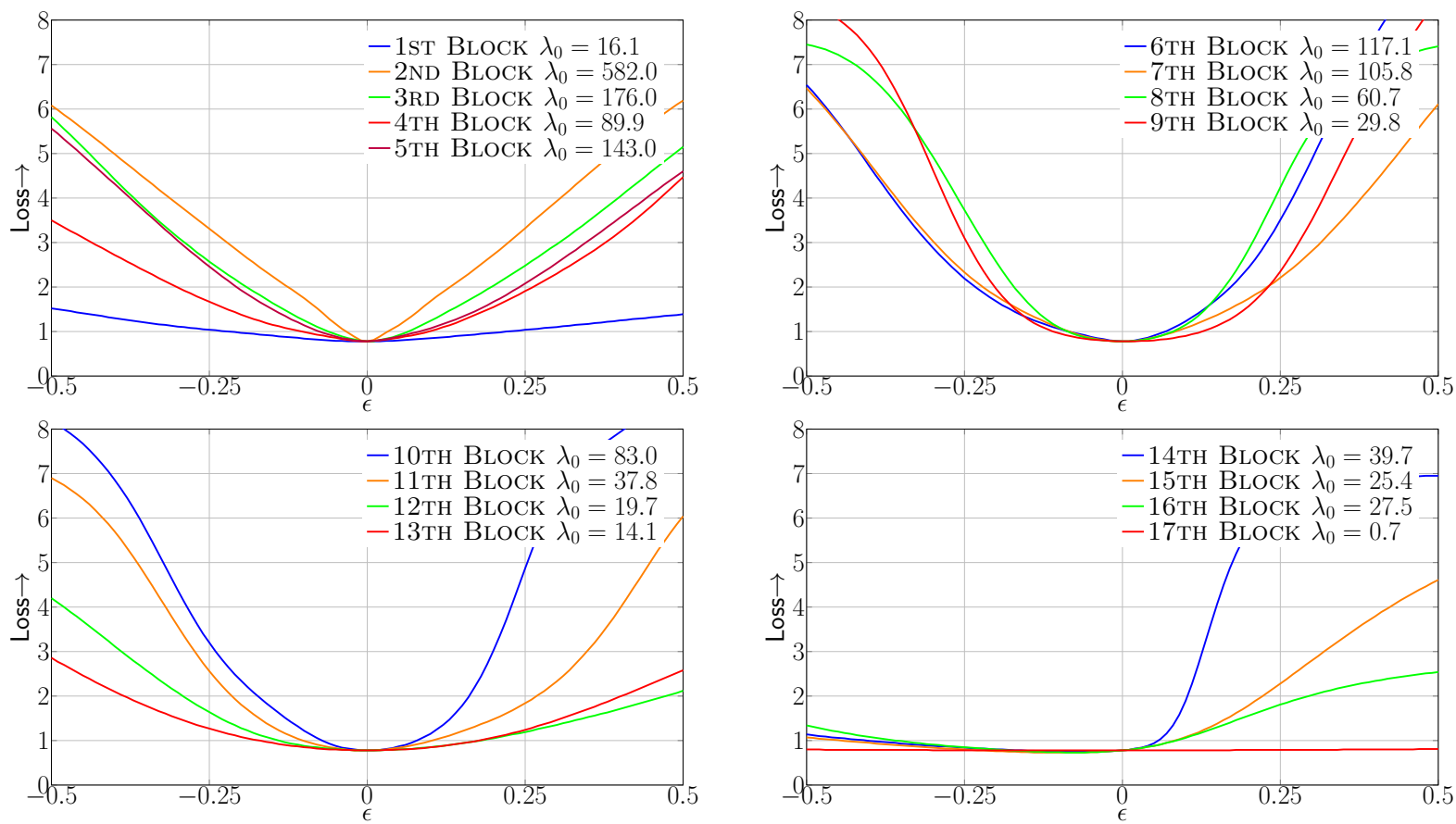

Fig. 3: 1-D loss landscape of all blocks of Inception-V3 on ImageNet along the first dominant eigenvector of the Hessian. Here $\epsilon$ is the scalar that perturbs the parameters of the corresponding block along the first dominant eigenvectors.

Table I: Quantization results of ResNet20 on Cifar-10. We abbreviate quantization bits used for weights as "w-bits," activations as "a-bits," testing accuracy as "Acc," and compression ratio of weights/activations as "W-Comp/A-Comp." Furthermore, we show results without using Hessian information ("Direct"), as well as other state-of-the-art methods [43], [2], [40]. In particular, we compare with the recent DNAS approach of [36]. Our method achieves similar testing performance with significantly higher compression (especially in activations). Here "MP" refers to mixed-precision quantization, where we report the lowest bits used for weights and activations. Also note that [43], [2], [40] use 8-bit for first and last layers. The exact per-layer configuration for mixed-precision quantization of HAWQ is presented in appendix.

\begin{tabular}{lccccc}
\hline Quantization & w-bits & a-bits & Acc & W-Comp & A-Comp \\
\hline Baseline & 32 & 32 & 92.37 & $1.00 \times$ & $1.00 \times$ \\
\hline Dorefa [43] & 2 & 2 & 88.20 & $16.00 \times$ & $16.00 \times$ \\
Dorefa [43] & 3 & 3 & 89.90 & $10.67 \times$ & $10.67 \times$ \\
PACT [2] & 2 & 2 & 89.70 & $16.00 \times$ & $16.00 \times$ \\
PACT [2] & 3 & 3 & 91.10 & $10.67 \times$ & $10.67 \times$ \\
LQ-Nets [40] & 2 & 2 & 90.20 & $16.00 \times$ & $16.00 \times$ \\
LQ-Nets [40] & 3 & 3 & 91.60 & $10.67 \times$ & $10.67 \times$ \\
LQ-Nets [40] & 2 & 32 & 91.80 & $16.00 \times$ & $1.00 \times$ \\
LQ-Nets [40] & 3 & 32 & 92.00 & $10.67 \times$ & $1.00 \times$ \\
DNAS [36] & 1 мР & 32 & 92.00 & $16.60 \times$ & $1.00 \times$ \\
DNAS [36] & 1 мР & 32 & $\mathbf{9 2 . 7 2}$ & $11.60 \times$ & $1.00 \times$ \\
\hline Direct & 2 мР & 4 & 90.34 & $16.00 \times$ & $8.00 \times$ \\
HAWQ & 2 мР & 4 & $\mathbf{9 2 . 2 2}$ & $13.11 \times$ & $8.00 \times$ \\
\hline
\end{tabular}

for Inception-V3, ResNet50, and SqueezeNext quantization on ImageNet. See appendix for details regarding the training procedure and hyper-parameters used.

a) Cifar-10: After computing the eigenvalues of block Hessian (shown in Figure 1), we compute the weighted sensitivity metric of Eq. 5, along with $\Omega_{i}$ based on Eq. 6 We then perform the quantization based on HAWQ algorithm. Results are shown in Table I]

For comparison, we test the quantization performance without using the Hessian information, which we refer to as "Direct" method, as well as other methods in the literature including Dorefa [43], PACT [2], LQ-Net [40], and DNAS [36], as shown in Table I

For methods that use Mixed-Precision (MP), we report the lowest bits used for weights ("w-bits"), and activations ("abits").

The Direct method achieves good compression, but it results in $2.03 \%$ accuracy drop, as shown in Table I Furthermore, comparison with other state-of-the-art shows a similar trend. There have been several methods proposed in the literature to address this reduction, with the latest method introduced in [40], where a learnable quantization method is used. As one can see, LQ-Nets results in $0.77 \%$ accuracy degradation with $10.67 \times$ compression ratio, whereas HAWQ has only $0.15 \%$ accuracy drop with $13.11 \times$ compression. Moreover, HAWQ achieves similar accuracy as compared to DNAS [36] but with $8 \times$ higher compression ratio for activations.

b) ImageNet: Here, we test the HAWQ method for quantizing Inception-V3 [34] on ImageNet. Inception-V3 is appealing for efficient hardware implementation, as it does not 
use any residual connections. Such non-linear structures create dependencies that may be very difficult to optimize for fast inference [37]. As before, we first compute the block Hessian eigenvalues, which are reported in Figure 1, and then compute the corresponding weighted sensitivity metric. We also plot the 1D loss landscape of all Inception-V3 blocks in Figure 3

We report the quantization results in Table II, where as before we compare with a direct quantization, as well as recently proposed "Integer-Only" [16], and RVQuant methods [26]. Direct quantization of Inception-V3 (i.e., without use of secondorder information), results in $7.69 \%$ accuracy degradation. Using the approach proposed in [16] results in more than $2 \%$ accuracy drop, even though it uses higher bit precision. However, HAWQ results in a generalization gap of $2 \%$ with a compression ratio of $12.04 \times$, both of which are better than previous work [16], [26] ${ }^{2}$

We also compare with Deep Compression [8] and the AutoML based method of HAQ, which has been recently introduced [35]. We compare our HAWQ results with their ResNet50 quantization, as shown in Table III. HAWQ achieves higher top-1 accuracy of $75.48 \%$ with a model size of $7.96 \mathrm{MB}$, whereas the AutoML based HAQ method has a top- 1 of $75.30 \%$ even with $16 \%$ larger model size of $9.22 \mathrm{MB}$.

Furthermore, we apply HAWQ to quantize SqueezeNext [7] on ImageNet. We choose the wider SqueezeNext model which has a baseline accuracy of $69.38 \%$ with 2.5 million parameters (10.1MB in single precision). We are able to quantize this model to uniform 8-bit precision, with just $0.04 \%$ top-1 accuracy drop. Direct quantization of SqueezeNext (i.e., without use of secondorder information), results in $3.98 \%$ accuracy degradation. HAWQ results in an unprecedented $1 \mathrm{MB}$ model size, with only $1.36 \%$ top- 1 accuracy drop. The significance of this result is that it allows deployment of the whole model on-chip or on hardwares with very limited memory and power constraints.

Table II: Quantization results of Inception-V3 on ImageNet. We abbreviate quantization bits used for weights as "w-bits," activations as "a-bits," top-1 testing accuracy as "Top-1," and weight compression ratio as "W-Comp." Furthermore, we compare HAWQ with direct quantization method without using Hessian ("Direct") and Integer-Only [16]. Here "MP" refers to mixed-precision quantization. We report the exact per-layer configuration for mixed-precision quantization in appendix. Compared to [16], [26], we achieve higher compression ratio with higher testing accuracy.

\begin{tabular}{lcccccc}
\hline Method & w-bits & a-bits & Top-1 & W-Comp & Size(MB) \\
\hline Baseline & 32 & 32 & 77.45 & $1.00 \times$ & 91.2 \\
\hline Integer-Only [16] & 8 & 8 & 75.40 & $4.00 \times$ & 22.8 \\
Integer-Only [16] & 7 & 7 & 75.00 & $4.57 \times$ & 20.0 \\
RVQuant [26] & 3 мР & 3 мP & 74.14 & $10.67 \times$ & 8.55 \\
\hline Direct & 2 мР & 4 мР & 69.76 & $15.88 \times$ & 5.74 \\
HAWQ & 2 мР & 4 мP & $\mathbf{7 5 . 5 2}$ & $12.04 \times$ & $\mathbf{7 . 5 7}$ \\
\hline
\end{tabular}

\footnotetext{
${ }^{2}$ We should emphasize here that the work of [16] uses integer arithmetic, and it is not completely fair to compare their results with ours.
}

Table III: Quantization results of ResNet50 on ImageNet. We show results of state-of-the-art methods [43], [2], [40], [8]. In particular, we also compare with the recent AutoML approach of [35]. Compared to [35], we achieve higher compression ratio with higher testing accuracy. Also note that [43], [2], [40] use 8-bit for first and last layers.

\begin{tabular}{lccccc}
\hline Method & w-bits & a-bits & Top-1 & W-Comp & Size(MB) \\
\hline Baseline & 32 & 32 & 77.39 & $1.00 \times$ & 97.8 \\
\hline Dorefa [43] & 2 & 2 & 67.10 & $16.00 \times$ & 6.11 \\
Dorefa [43] & 3 & 3 & 69.90 & $10.67 \times$ & 9.17 \\
PACT [2] & 2 & 2 & 72.20 & $16.00 \times$ & 6.11 \\
PACT [2] & 3 & 3 & 75.30 & $10.67 \times$ & 9.17 \\
LQ-Nets [40] & 3 & 3 & 74.20 & $10.67 \times$ & 9.17 \\
Deep Comp. [8] & 3 & MP & 75.10 & $10.41 \times$ & 9.36 \\
HAQ [35] & MP & MP & 75.30 & $10.57 \times$ & 9.22 \\
\hline HAWQ & 2 мP & 4 мP & $\mathbf{7 5 . 4 8}$ & $12.28 \times$ & $\mathbf{7 . 9 6}$ \\
\hline
\end{tabular}

Table IV: Quantization results of SqueezeNext on ImageNet. We show a case where HAWQ is used to achieved uniform quantization to 8 bits for both weights and activations, with an accuracy similar to ResNet18. We also show a case with mixed precision, where we compress SqueezeNext to a model with just $1 M B$ size with only $1.36 \%$ accuracy degradataion. Furthermore, we compare HAWQ with direct quantization method without using Hessian ("Direct").

\begin{tabular}{lccccc}
\hline Method & w-bits & a-bits & Top-1 & W-Comp & Size(MB) \\
\hline Baseline & 32 & 32 & 69.38 & $1.00 \times$ & 10.1 \\
ResNet18 [27] & 32 & 32 & 69.76 & $1.00 \times$ & 44.7 \\
\hline HAWQ & 8 & 8 & $\mathbf{6 9 . 3 4}$ & $4.00 \times$ & 2.53 \\
Direct & 3 мP & 8 & $\mathbf{6 5 . 3 9}$ & $9.04 \times$ & 1.12 \\
HAWQ & 3 мP & 8 & $\mathbf{6 8 . 0 2}$ & $9.25 \times$ & $\mathbf{1 . 0 9}$ \\
\hline
\end{tabular}

\section{Ablation Study}

Here we discuss the ablation study for the HAWQ. The HAWQ method has two main steps: (i) relative precision order for different blocks using second-order information, and (ii) relative order for fine-tuning these blocks. Below we discuss the ablation study for each step separately.

\section{A. Hessian AWare Mixed Precision Quantization}

We first discuss the ablation study for step (i), where the quantization precision is chosen based on Eq. 5. As discussed above, blocks with higher values of $S_{i}$ are assigned higher quantization precision, and vice versa for layers with relatively lower values of $S_{i}$. For the ablation study we reverse this order and avoid performing the block-wise fine-tuning of step (ii) so we can isolate step (i). Instead of the fine-tuning phase, we re-train the whole network at once after the quantization is performed. The results are shown in Figure 4, where we perform 50 epochs of fine-tuning using Inception-V3 on ImageNet. As one can see, HAWQ results in significantly better accuracy $(74.26 \%$ as compared to $66.72 \%)$ than the reverse method (labeled as "HAWQ-Reverse-Precision"). This is despite the 


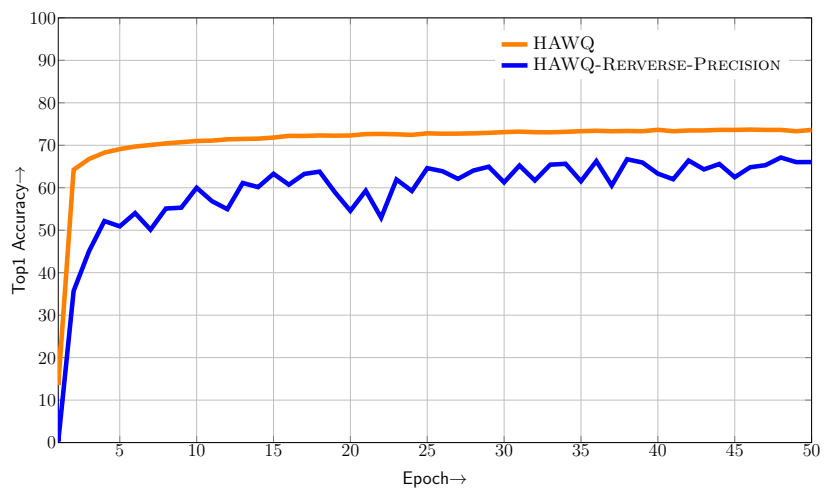

Fig. 4: Accuracy recovery from Hessian aware mixed precision quantization versus HAWQ-Reverse-Precision quantization. Here, we show top-1 accuracy of quantized Inception-V3 on ImageNet. HAWQ-Reverse-Precision achieves $66.72 \%$ (compression-ratio 7.2) top-1 accuracy, while our HAWQ method achieves $74.36 \%$ (compression-ratio 12.0) top-1 accuracy (7.64\% better) with a higher convergence speed (30 epochs v.s. 50 epochs of HAWQ-Reverse-Precision).

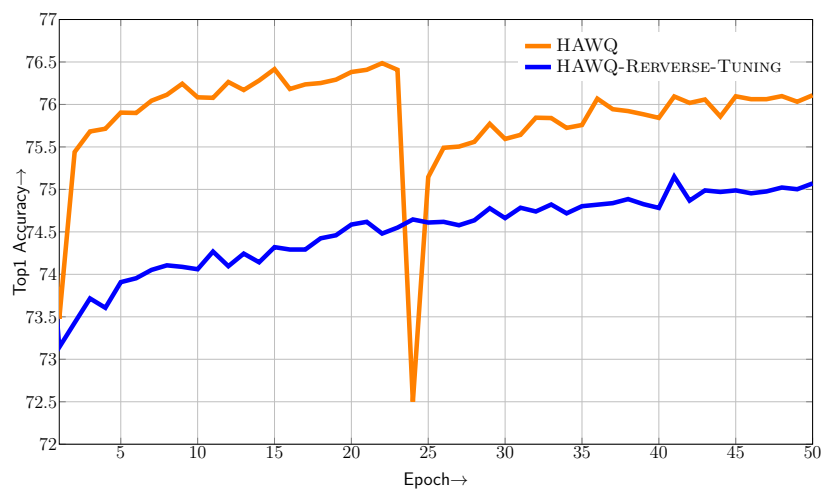

Fig. 5: Effectiveness of Hessian aware block-wise quantization. Here, HAWQ shows the quantization process based on the descending order of $\Omega_{i}$ for Inception-V3 with Hessian aware quantization order. HAWQ-Reverse-Tuning shows the quantization process of Inception-V3 with a reverse order. Note that HAWQ finishes the fine-tuning of this block in just 25 epochs and switches to fine-tuning another block, whereas HAWQ-Reverse-Tuning takes 50 epochs for this block, before converging to sub-optimal top-1.

fact that the latter approach only has a compression ratio of $7.2 \times$, whereas HAWQ has a compression ratio of $12.0 \times$.

Another interesting observation is that the convergence speed of the Hessian aware approach is significantly faster than the reverse method. Here, HAWQ converges in about 30 epochs, whereas the HAWQ-Reverse-Precision case takes 50 epochs before converging to a sub-optimal value (Figure 47).

\section{B. Block-Wise Fine-Tuning}

Here we perform the ablation study for the Hessian based fine-tuning part of HAWQ. The block-wise fine tuning is performed based on $\Omega_{i}$ (Eq. 6) of each block. The blocks are fine-tuned based on the descending order of $\Omega_{i}$. Similar to the above, we compare the quantization performance when a reverse ordering is used (i.e., we use the ascending order of $\Omega_{i}$ and refer to this as "HAWQ-Reverse-Tuning").

We test this ablation study using Inception-V3 on ImageNet, as shown in Figure 5. As one can see, the fine-tuning for HAWQ method quickly converges in just 25 epochs, allowing it to switch to fine-tuning the next block. However, "HAWQReverse-Tuning" takes more than 50 epochs to converge for this block.

\section{CONCLUSIONS}

We have introduced HAWQ, a new quantization method for neural network training. Our method is based on exploiting second-order (Hessian) information to systematically select both quantization precision as well as the order for block-wise fine-tuning. We performed an ablation study for both the relative quantization bit-order for different blocks, as well as the finetuning order. We showed that HAWQ can achieve good testing performance with high compression-ratio, as compared to stateof-the-art. In particular, we showed results for ResNet20 on Cifar-10, where we can achieve similar testing performance as [36], but with $8 \times$ higher compression ratio for activations. We also showed results for Inception-V3 on ImageNet, for which we showed ultra low precision quantization results with 2-bit for weights and 4-bit for activations, with only $1.93 \%$ accuracy drop. For ResNet50 model, our approach results in higher accuracy of $75.48 \%$ with smaller model size of $7.96 \mathrm{MB}$, as compared to HAQ method with top-1 of $75.30 \%$ and 9.22MB [35]. Furthermore, our method applied to SqueezeNext can result in an unprecedented $1 \mathrm{MB}$ model size with 68.02 top-1 accuracy on ImageNet.

Limitations and Future Work. We believe it is critical for every work to clearly state its limitations, especially in this area. An important limitation is that computing the secondorder information adds some computational overhead. However, we only need to compute the top eigenvalue of the Hessian, which can be found using the matrix-free method presented in Algorithm 1 (The total computational overhead is equivalent to about 20 gradient back-propogations to compute top Hessian eigenvalue of each block). Another limitation is that in this work we solely focused on image classification, but it would be interesting to see how HAWQ would perform for more complex tasks such as segmentation, object detection, or natural language processing. Furthermore, one has to consider that implementation of a $\mathrm{NN}$ with mixed-precision inference for embedded processors is not as straightforward as the case with uniform quantization precision. Practical solutions have been proposed in recent works [32]. Another limitation is that we can only determine the relative ordering for quantization precision, and not the absolute value of the bits. However, the search space for this is significantly smaller than the original exponential complexity. Finally, even though we showed benefits of HAWQ as compared to DNAS [36] or HAQ [35], it may be possible to combine these methods for more efficient AutoML search. We leave this as part of future work. 


\section{ACKNOWLEDGMENTS}

This work was supported by a gracious fund from Intel corporation, Berkeley Deep Drive (BDD), and Berkeley AI Research (BAIR) sponsors. We would like to thank the Intel VLAB team for providing us with access to their computing cluster. We also gratefully acknowledge the support of NVIDIA Corporation for their donation of two Titan Xp GPU used for this research. We would also like to acknowledge ARO, DARPA, NSF, and ONR for providing partial support of this work.

\section{REFERENCES}

[1] K. Asanovic and N. Morgan. Experimental determination of precision requirements for back-propagation training of artificial neural networks. International Computer Science Institute, 1991.

[2] J. Choi, Z. Wang, S. Venkataramani, P. I.-J. Chuang, V. Srinivasan, and K. Gopalakrishnan. Pact: Parameterized clipping activation for quantized neural networks. arXiv preprint arXiv:1805.06085, 2018.

[3] F. Chollet. Xception: Deep learning with depthwise separable convolutions. In Proceedings of the IEEE conference on computer vision and pattern recognition, pages 1251-1258, 2017.

[4] M. Courbariaux, Y. Bengio, and J.-P. David. Binaryconnect: Training deep neural networks with binary weights during propagations. In Advances in neural information processing systems, pages 3123-3131, 2015.

[5] E. L. Denton, W. Zaremba, J. Bruna, Y. LeCun, and R. Fergus. Exploiting linear structure within convolutional networks for efficient evaluation. In Advances in neural information processing systems, pages 1269-1277, 2014.

[6] Y. Dong, R. Ni, J. Li, Y. Chen, J. Zhu, and H. Su. Learning accurate lowbit deep neural networks with stochastic quantization. British Machine Vision Conference, 2017.

[7] A. Gholami, K. Kwon, B. Wu, Z. Tai, X. Yue, P. Jin, S. Zhao, and K. Keutzer. Squeezenext: Hardware-aware neural network design. Workshop paper in CVPR, 2018.

[8] S. Han, H. Mao, and W. J. Dally. Deep compression: Compressing deep neural networks with pruning, trained quantization and huffman coding. International Conference on Learning Representations, 2016.

[9] S. Han, J. Pool, J. Tran, and W. Dally. Learning both weights and connections for efficient neural network. In Advances in neural information processing systems, pages 1135-1143, 2015.

[10] K. He, X. Zhang, S. Ren, and J. Sun. Deep residual learning for image recognition. In Proceedings of the IEEE conference on computer vision and pattern recognition, pages 770-778, 2016

[11] G. Hinton, O. Vinyals, and J. Dean. Distilling the knowledge in a neural network. Workshop paper in NIPS, 2014.

[12] S. Hochreiter and J. Schmidhuber. Flat minima. Neural Computation, 9(1):1-42, 1997

[13] A. G. Howard, M. Zhu, B. Chen, D. Kalenichenko, W. Wang, T. Weyand, M. Andreetto, and H. Adam. Mobilenets: Efficient convolutional neural networks for mobile vision applications. arXiv preprint arXiv:1704.04861 2017.

[14] I. Hubara, M. Courbariaux, D. Soudry, R. El-Yaniv, and Y. Bengio. Quantized neural networks: Training neural networks with low precision weights and activations. The Journal of Machine Learning Research, 18(1):6869-6898, 2017.

[15] F. N. Iandola, S. Han, M. W. Moskewicz, K. Ashraf, W. J. Dally, and K. Keutzer. Squeezenet: Alexnet-level accuracy with 50x fewer parameters andi $0.5 \mathrm{mb}$ model size. arXiv preprint arXiv:1602.07360, 2016.

[16] B. Jacob, S. Kligys, B. Chen, M. Zhu, M. Tang, A. Howard, H. Adam, and D. Kalenichenko. Quantization and training of neural networks for efficient integer-arithmetic-only inference. In Proceedings of the IEEE Conference on Computer Vision and Pattern Recognition, pages 2704-2713, 2018

[17] R. Krishnamoorthi. Quantizing deep convolutional networks for efficient inference: A whitepaper. arXiv preprint arXiv:1806.08342, 2018.

[18] A. Krizhevsky, I. Sutskever, and G. E. Hinton. Imagenet classification with deep convolutional neural networks. In Advances in neural information processing systems, pages 1097-1105, 2012.

[19] Y. LeCun, L. Bottou, Y. Bengio, P. Haffner, et al. Gradient-based learning applied to document recognition. Proceedings of the IEEE, 86(11):2278-2324, 1998.
[20] H. Li, A. Kadav, I. Durdanovic, H. Samet, and H. P. Graf. Pruning filters for efficient convnets. arXiv preprint arXiv:1608.08710, 2016.

[21] N. Ma, X. Zhang, H.-T. Zheng, and J. Sun. Shufflenet v2: Practical guidelines for efficient $\mathrm{cnn}$ architecture design. In Proceedings of the European Conference on Computer Vision (ECCV), pages 116-131, 2018.

[22] H. Mao, S. Han, J. Pool, W. Li, X. Liu, Y. Wang, and W. J. Dally. Exploring the regularity of sparse structure in convolutional neural networks. Workshop paper in CVPR, 2017.

[23] J. Martens. Deep learning via hessian-free optimization. In ICML, volume 27, pages 735-742, 2010.

[24] D. Miyashita, E. H. Lee, and B. Murmann. Convolutional neural networks using logarithmic data representation. arXiv preprint arXiv:1603.01025

[25] P. Molchanov, S. Tyree, T. Karras, T. Aila, and J. Kautz. Pruning convolutional neural networks for resource efficient inference. arXiv preprint arXiv:1611.06440, 2016

[26] E. Park, S. Yoo, and P. Vajda. Value-aware quantization for training and inference of neural networks. In Proceedings of the European Conference on Computer Vision (ECCV), pages 580-595, 2018.

[27] A. Paszke, S. Gross, S. Chintala, G. Chanan, E. Yang, Z. DeVito, Z. Lin, A. Desmaison, L. Antiga, and A. Lerer. Automatic differentiation in pytorch. 2017.

[28] M. Rastegari, V. Ordonez, J. Redmon, and A. Farhadi. Xnor-net: Imagenet classification using binary convolutional neural networks. In European Conference on Computer Vision, pages 525-542. Springer, 2016.

[29] J. Rissanen. Modeling by shortest data description. Automatica, 14(5):465471, 1978.

[30] A. Romero, N. Ballas, S. E. Kahou, A. Chassang, C. Gatta, and Y. Bengio Fitnets: Hints for thin deep nets. arXiv preprint arXiv:1412.6550, 2014

[31] M. Sandler, A. Howard, M. Zhu, A. Zhmoginov, and L.-C. Chen Mobilenetv2: Inverted residuals and linear bottlenecks. In Proceedings of the IEEE Conference on Computer Vision and Pattern Recognition, pages 4510-4520, 2018.

[32] H. Sharma, J. Park, N. Suda, L. Lai, B. Chau, V. Chandra, and H. Esmaeilzadeh. Bit fusion: Bit-level dynamically composable architecture for accelerating deep neural networks. In Proceedings of the 45th Annual International Symposium on Computer Architecture, pages 764-775. IEEE Press, 2018

[33] K. Simonyan and A. Zisserman. Very deep convolutional networks for large-scale image recognition. In International Conference on Learning Representations, 2015.

[34] C. Szegedy, V. Vanhoucke, S. Ioffe, J. Shlens, and Z. Wojna. Rethinking the inception architecture for computer vision. In Proceedings of the IEEE conference on computer vision and pattern recognition, pages 2818-2826, 2016.

[35] K. Wang, Z. Liu, Y. Lin, J. Lin, and S. Han. HAQ: Hardware-aware automated quantization. In Proceedings of the IEEE conference on computer vision and pattern recognition, 2019.

[36] B. Wu, Y. Wang, P. Zhang, Y. Tian, P. Vajda, and K. Keutzer. Mixed precision quantization of convnets via differentiable neural architecture search. arXiv preprint arXiv:1812.00090, 2018.

[37] Y. Yang, Q. Huang, B. Wu, T. Zhang, L. Ma, G. Gambardella, M. Blott, L. Lavagno, K. Vissers, J. Wawrzynek, et al. Synetgy: Algorithmhardware co-design for convnet accelerators on embedded fpgas. In Proceedings of the 2019 ACM/SIGDA International Symposium on FieldProgrammable Gate Arrays, pages 23-32. ACM, 2019.

[38] Z. Yao, A. Gholami, K. Keutzer, and M. Mahoney. Large batch size training of neural networks with adversarial training and second-order information. arXiv preprint arXiv:1810.01021, 2018.

[39] Z. Yao, A. Gholami, Q. Lei, K. Keutzer, and M. W. Mahoney. Hessianbased analysis of large batch training and robustness to adversaries. Advances in Neural Information Processing Systems, 2018.

[40] D. Zhang, J. Yang, D. Ye, and G. Hua. LQ-Nets: Learned quantization for highly accurate and compact deep neural networks. In The European Conference on Computer Vision (ECCV), September 2018.

[41] X. Zhang, X. Zhou, M. Lin, and J. Sun. Shufflenet: An extremely efficient convolutional neural network for mobile devices. In Proceedings of the IEEE Conference on Computer Vision and Pattern Recognition, pages 6848-6856, 2018

[42] A. Zhou, A. Yao, Y. Guo, L. Xu, and Y. Chen. Incremental network quantization: Towards lossless cnns with low-precision weights. International Conference on Learning Representations, 2017.

[43] S. Zhou, Y. Wu, Z. Ni, X. Zhou, H. Wen, and Y. Zou. Dorefa-net: Training low bitwidth convolutional neural networks with low bitwidth gradients. arXiv preprint arXiv:1606.06160, 2016.

[44] Y. Zhou, S.-M. Moosavi-Dezfooli, N.-M. Cheung, and P. Frossard Adaptive quantization for deep neural network. In Thirty-Second AAAI Conference on Artificial Intelligence, 2018. 


\section{APPENDIX}

Here, we provide additional experimental results as well as quantization details for the neural networks that we tested.

- In $\S$ VII-A we discuss the fine-tuning details.

- In $\S$ VII-B we present extra results for 3D plots for loss landscape of different blocks of ResNet20 and Inception-V3 as well as exemplary results showing distribution of $\Omega_{i}$ in Eq. 6

- In $\S$ VII-C we show the exact bit-precision used for different blocks of ResNet20 on Cifar-10 as well as Inception-V3 on ImageNet.

\section{A. Fine-tuning details}

The results were tested on two classification datasets of Cifar-10 and ImageNet:

a) Cifar-10: This is a classification dataset with 10 classes consisting of 50,000 training images and 10,000 test images of size $3 \times 32 \times 32$. We used pre-trained ResNet 20 model and performed quantization on this model in PyTorch framework. We follow the same learning rate policy as the baseline (i.e., decaying learning rate from 0.1 to 0.0001 ).

b) ImageNet: This is a classification problem with 1000 classes consisting of more than 1.2 million training images and 50,000 validation images of size $3 \times 224 \times 224$ on SqueezeNext and ResNet50, and $3 \times 299 \times 299$ on Inception-V3. (i) We used pre-trained Inception-V3 model and used a fixed learning rate of 0.0002 for fine-tuning of each block. (ii) We used pre-trained ResNet50 model and used a fixed learning rate of 0.0001 for fine-tuning of each block. (iii) We used pre-trained SqueezeNext and used a fixed learning rate of 0.0001 for fine-tuning of each block. All experiments were performed on PyTorch framework. As for data augmentation, we used standard random crop, resizing and horizontal flip in all experiments.

\section{B. Extra results}

In Table $\mathrm{V}$, we show how $\Omega_{i}$ changes as a function of quantization precision. In Figure 6, we plot the rest surface visualization of ResNet20 on Cifar-10. And in Figure 7, we plot the rest surface visualization of Inception-V3 on ImageNet.

Table V: Here we show how $\Omega_{i}$ changes as a function of target weight bit precision. Results are computed for ResNet20 on Cifar-10.

\begin{tabular}{lcccccc}
\hline Block & Precision & 8-bit & 6-bit & 4-bit & 3-bit & 2-bit \\
\hline Block 3 & & & & & & \\
Block 5 & 0.03 & 0.52 & 9.25 & 41.9 & 191 \\
Block 8 & 0.05 & 0.81 & 14.0 & 65.1 & 309 \\
\hline
\end{tabular}

\section{Mixed-precision details}

In this section, we give the details about how we separate blocks and details about weight/activation precision of each individual block. We show the exact bit-precision used for different blocks of ResNet20 (Table VI) on Cifar-10 as well as Inception-V3 (Table VII).

Table VI: Block seperation and final block precision of ResNet20 on Cifar-10. Here we abbreviate convolutional layer as "Conv," fully connected layer as "FC."

\begin{tabular}{cccccc}
\hline Block & Layer(s) & Layer Type & Parameter Size & Weight bit & Activation bit \\
\hline Block 0 & Layer 0 & Conv & $4.32 \mathrm{e} 2$ & 8 & 8 \\
\hline Block 1 & Layer 1-2 & Conv & $4.61 \mathrm{e} 3$ & 6 & 4 \\
\hline Block 2 & Layer 3-4 & Conv & $4.61 \mathrm{e} 3$ & 6 & 4 \\
\hline Block 3 & Layer 5-6 & Conv & $4.61 \mathrm{e} 3$ & 8 & 4 \\
\hline Block 4 & Layer 7-8 & Conv & $1.38 \mathrm{e} 4$ & 3 & 4 \\
\hline Block 5 & Layer 9-10 & Conv & $1.84 \mathrm{e} 4$ & 3 & 4 \\
\hline Block 6 & Layer 11-12 & Conv & $1.84 \mathrm{e} 4$ & 3 & 4 \\
\hline Block 7 & Layer 13-14 & Conv & $5.53 \mathrm{e} 4$ & 2 & 4 \\
\hline Block 8 & Layer 15-16 & Conv & $7.37 \mathrm{e} 4$ & 2 & 4 \\
\hline Block 9 & Layer 17-18 & Conv & $7.37 \mathrm{e} 4$ & 2 & 4 \\
\hline Block 10 & Layer 19 & FC & $6.40 \mathrm{e} 2$ & 3 & 8 \\
\hline
\end{tabular}



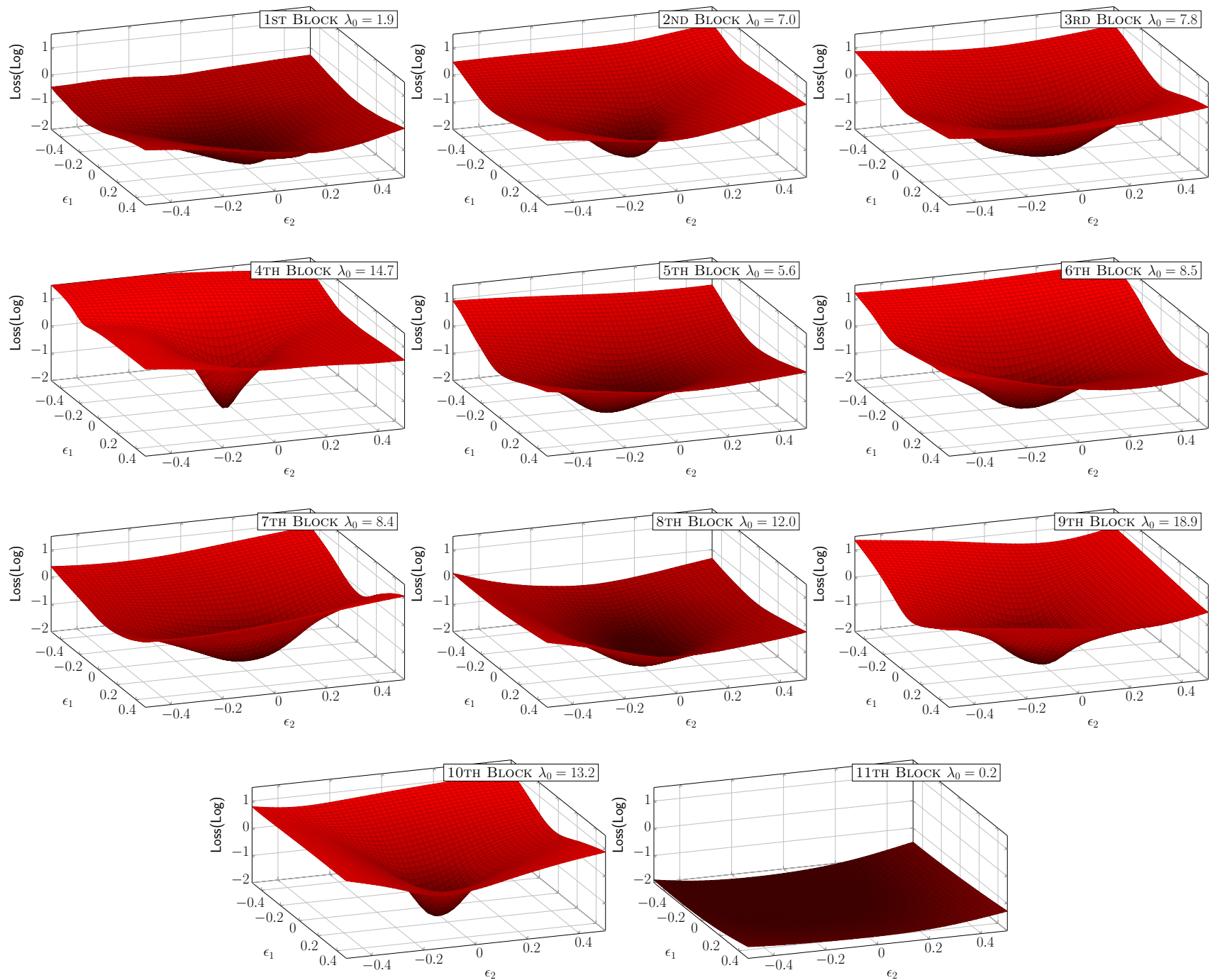

Fig. 6: 3-D loss landscape of all blocks of ResNet20 on Cifar-10 along the first two dominant eigenvectors of the Hessian. Here $\epsilon_{1}, \epsilon_{2}$ are scalars that perturb the parameters of the corresponding block along the first and second dominant eigenvectors. The corresponding eigenvalue distribution for different blocks is shown in Figure 1 . 

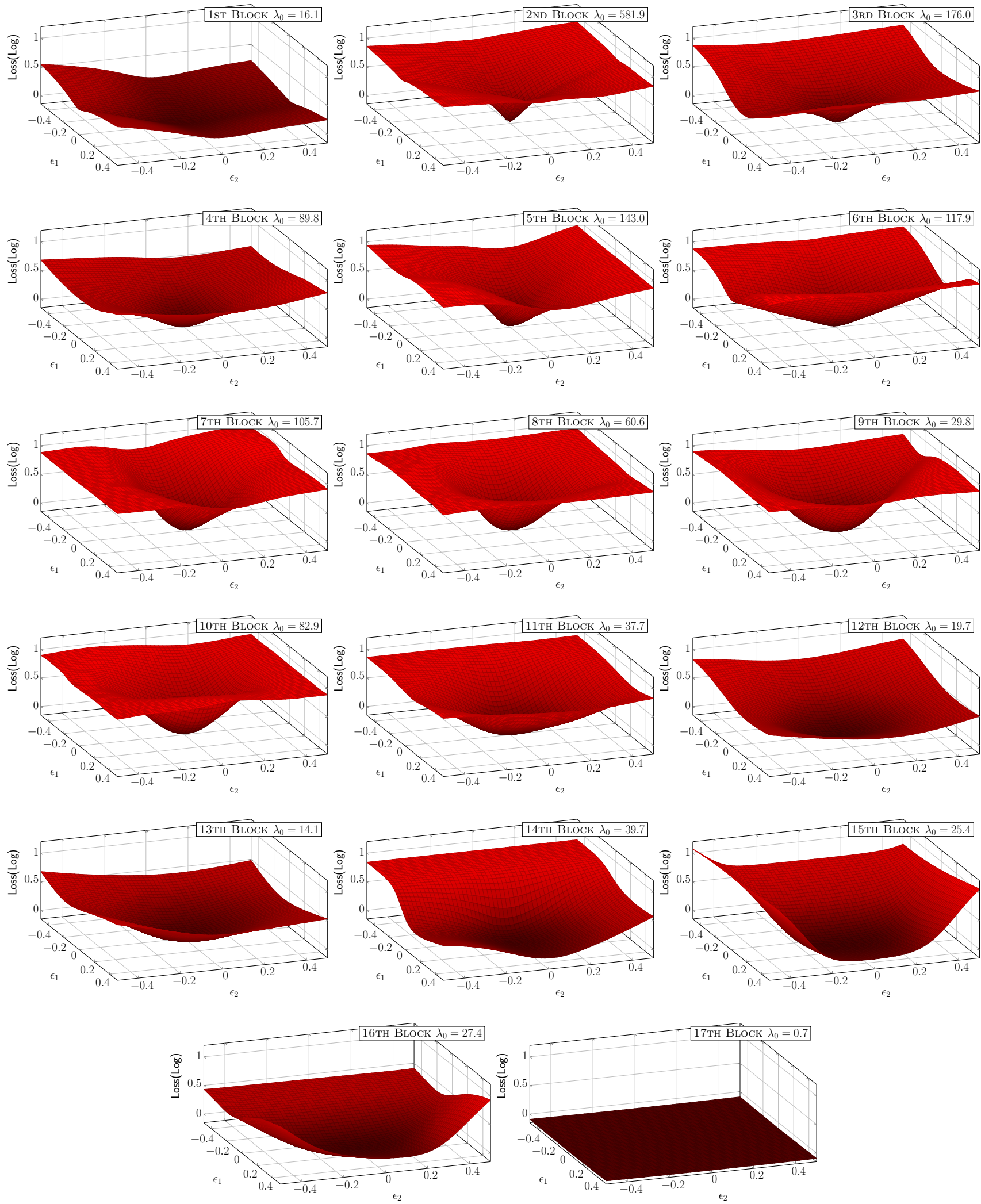

Fig. 7: 3-D loss landscape of all blocks of InceptionV3 on ImageNet along the first two dominant eigenvectors of the Hessian. Here $\epsilon_{1}, \epsilon_{2}$ are scalars that perturb the parameters of the corresponding block along the first and second dominant eigenvectors. The corresponding eigenvalue distribution for different blocks is shown in Figure 1 . 
Table VII: Block seperation and final block precision of Inception-V3 on ImageNet. Here we abbreviate convolutional layer as "Conv," fully connected layer as "FC."

\begin{tabular}{cccccc}
\hline Block & Layer(s) & Layer Type & Parameter Size(M) & Weight bit & Activation bit \\
\hline Block 0 & Layer 0 & Conv & $8.64 \mathrm{e}-4$ & 6 & 6 \\
\hline Block 1 & Layer 1 & Conv & $9.22 \mathrm{e}-3$ & 6 & 6 \\
\hline Block 2 & Layer 2 & Conv & $1.84 \mathrm{e}-2$ & 4 & 6 \\
\hline Block 3 & Layer 3 & Conv & $5.12 \mathrm{e}-3$ & 4 & 6 \\
\hline Block 4 & Layer 4 & Conv & 0.14 & 4 & 6 \\
\hline Block 5 & Layer 5-11 & Conv & 0.25 & 4 & 4 \\
\hline Block 6 & Layer 12-18 & Conv & 0.28 & 4 & 4 \\
\hline Block 7 & Layer 19-25 & Conv & 0.28 & 4 & 4 \\
\hline Block 8 & Layer 26-29 & Conv & 1.15 & 2 & 4 \\
\hline Block 9 & Layer 30-39 & Conv & 1.29 & 4 & 4 \\
\hline Block 10 & Layer 40-49 & Conv & 1.69 & 4 & 4 \\
\hline Block 11 & Layer 50-59 & Conv & 1.69 & 4 & 4 \\
\hline Block 12 & Layer 60-69 & Conv & 2.14 & 4 & 4 \\
\hline Block 13 & Layer 70-75 & Conv & 1.70 & 2 & 4 \\
\hline Block 14 & Layer 76-84 & Conv & 5.04 & 2 & 4 \\
\hline Block 15 & Layer 85-93 & Conv & 6.07 & 2 & 4 \\
\hline Block 16 & Layer 94 & FC & 2.05 & 2 & 4 \\
\hline
\end{tabular}

